\section{Revisão dos estudos de avaliação da ocorrência de eventos adversos em hospitais}

\section{Review of studies on the assessment of adverse events in hospitals}

Walter Mendes ${ }^{1}$

Cláudia Travassos ${ }^{2}$

Mônica Martins ${ }^{1}$

José Carvalho de Noronha ${ }^{2}$

${ }^{1}$ Escola Nacional de Saúde Pública - Fundação Oswaldo Cruz

${ }^{2}$ Centro de Informações de Ciência e Tecnologia - Fundação Oswaldo Cruz

Correspondência: Walter Mendes. Escola de Governo em Saúde - Rua Leopoldo Bulhões, 1480 - $3 \circ$ andar - Manguinhos, 21041-210 Rio de Janeiro - RJ.E-mail:wmendes@ensp.fiocruz.br

\section{Resumo}

Objetivo: Rever os estudos sobre a ocorrência de eventos adversos em hospitais, com objetivo de avaliar as metodologias empregadas e os seus resultados. Metodologia: Revisão sistemática da literatura científica sobre avaliação da ocorrência de evento adverso em hospitais. Esta pesquisa incluiu apenas os estudos que empregaram na avaliação o método de revisão retrospectiva de prontuário do paciente. A análise dos estudos abordou a definição de evento adverso e de eventos adversos evitáveis, a incidência, a forma de aplicação do método de revisão retrospectiva; os critérios de avaliação empregados e as categorias de análise: área clínica, especialidade, local de ocorrência, momento da ocorrência do evento adverso, gravidade da lesão. Resultados: Foram identificados nove estudos publicados nos Estados Unidos da América, Austrália, Nova Zelândia, França, Inglaterra, Dinamarca e Canadá, que avaliaram, no total, 92063 prontuários. A partir da mudança do enfoque dos estudos, que evoluiu de um caráter médico legal para estudos de melhoria de qualidade, o evento adverso passou a ser definido como lesão [injury] não intencional que resultou em incapacidade [disability] temporária ou permanente e/ou prolongamento do tempo de permanência ou morte como conseqüência do cuidado prestado. A incidência de eventos adversos variou de 2,9 a 16,6 por 100 pacientes admitidos em hospitais nos estudos revisados. Conclusão: Em que pesem as diferenças nos critérios de avaliação e dos resultados nos nove estudos, eles permitem orientar o desenho da metodologia para mensurar um fenômeno cuja magnitude não é conhecida no Brasil.

Palavras-chave: Eventos adversos. Avaliação de serviços de saúde. Qualidade na assistência à saúde. Segurança do paciente. Erro médico. 


\section{Abstract}

Objective: To review studies on Adverse Events in hospitals, with a focus on methodologies and study results. Methodology: Systematic review of the scientific literature on the assessment of adverse events in hospitals. This review only included studies that applied the method of retrospective chart review in the assessment of adverse events. The review focused on the definition of adverse events and avoidable adverse event chart review methods; evaluation criteria and categories of analysis: specialty, clinical area, time and place of the event, severity and degree of incapacity. Results: Nine studies from the United States, Australia, New Zealand, France, England, Denmark and Canada reviewed 92,063 medical records. Initial studies were based on a medical and legal approach and evolved to a continuing quality improvement strategy. In the latter, adverse event is defined as an unintended injury resulting in temporary or permanent disability and/or prolonged length of stay as a consequence of health care management. The incidence of adverse events varied from 2.9 to 16.6 per 100 in patients in the studies reviewed. Conclusion: Despite differences in the criteria and results of the studies reviewed in this paper, all of them were directed at measuring a phenomenon whose magnitude is still unknown in our country.

Key words: Adverse events. Evaluation of health services. Quality in health care. Patient security. Medical error.

\section{Introdução}

Há duas décadas pesquisadores estudam de forma sistemática um fenômeno relacionado a problemas de qualidade nos serviços de saúde. Esse fenômeno - os eventos adversos (EAs) - atinge, nos países onde foi investigado, patamares alarmantes ${ }^{1}$. Estima-se que cerca de 100 mil pessoas morram em hospitais a cada ano, vítimas de EAs nos Estados Unidos da América (EUA). Essa alta incidência resulta em uma taxa de mortalidade, nos EUA, maior do que as atribuídas aos pacientes com AIDS, câncer de mama ou atropelamentos ${ }^{2}$.

A ocorrência crescente de casos documentados de EAs no cuidado à saúde tem provocado um debate sobre a segurança do paciente em âmbito internacional. Estudos sobre agravos causados pelo cuidado à saúde já vêm sendo divulgados há muitos anos. Em 1850, o médico húngaro Ignaz Semmelweiss estabeleceu a ligação entre a transmissão da infecção e a higiene da mão ${ }^{3}$. Em 1854, Florence Nightingale revolucionou a atenção hospitalar a partir da observação dos riscos para o paciente da má higiene nos hospitais ${ }^{4}$. No início do século XX, Ernest Codman, um cirurgião de Boston, apontou para a necessidade de avaliação rotineira dos resultados negativos na cirurgia para a melhoria da qualidade ${ }^{5}$. O reconhecimento de que a exposição ao sistema de saúde pode gerar danos, e não só proporcionar benefícios, foi descrito em 1955 por Barr como sendo o ônus inevitável que se paga pelos avanços tecnológicos da medicina, dando origem às enfermidades denominadas "doenças do progresso médico" 6 .

A ocorrência de EA representa também um grave prejuízo financeiro. No Reino Unido e na Irlanda do Norte, o prolongamento do tempo de permanência no hospital devido aos EAs custa cerca de dois bilhões de libras ao ano e o gasto do Sistema Nacional de Saúde com questões litigiosas associadas a EAs é de 400 milhões de libras ao ano. Nos EUA, os custos anuais provocados por EAs estão estimados entre 17 e 29 bilhões de dólares anuais ${ }^{1}$. 
Frente à magnitude do problema, a Organização Mundial de Saúde (OMS) criou, em 2002, um Grupo de Trabalho com o objetivo de estudar metodologias para avaliar de forma sistemática os riscos à segurança do paciente nos serviços de saúde. O relatório do GT da OMS ${ }^{1}$, apesar de descrever todos os métodos utilizados para analisar EAs, só comparou os resultados das avaliações baseadas em revisão retrospectiva de prontuários, com essa metodologia. O livro To Err is human ${ }^{2}$ também só faz menção a esse tipo de avaliação. A revisão retrospectiva de prontuário tem sido considerada o método de coleta de dados mais adequado (gold standard) para a avaliação de EAs, apesar de apresentar alto custo e de conter imperfeições?.

Mais recentemente as pesquisas têm se voltado para a elaboração de metodologia para a detecção de EAs usando meios eletrônicos ${ }^{7}$. Busca-se dessa forma desenvolver programas de fácil execução, que possam ser incorporados à rotina dos serviços. No entanto, as metodologias que empregam dados disponíveis em meios eletrônicos ainda não são factíveis de aplicação na maior parte dos países, inclusive o Brasil.

Mundialmente, a discussão da qualidade do cuidado à saúde volta-se cada vez mais para a questão dos EAs ${ }^{1-3,7-9}$. Entretanto, poucos são os estudos brasileiros sobre a ocorrência de $\mathrm{EAs}^{3}$, e os trabalhos realizados enfocaram causas específicas, como EAs provocados por medicamentos ou decorrentes de complicações de métodos terapêuticos cruentos.

Neste trabalho, realizou-se uma revisão sistemática da literatura em saúde pública sobre estudos voltados para quantificar a ocorrência de EAs em hospitais, limitando-se àqueles baseados na revisão retrospectiva de prontuário, metodologia mais utilizada em estudos de EAs e factível para avaliação dos hospitais brasileiros.

\section{Metodologia}

Na revisão sistemática foram selecionados os estudos baseados na revisão retrospectiva de prontuários que avaliaram a ocor- rência de EAs no conjunto das ações dos hospitais. Foram incluídos na revisão apenas os estudos centrados em hospitais que avaliaram todos os tipos de EAs e excluídos aqueles que se detiveram na avaliação da ocorrência de EAs em outras áreas de cuidado - por exemplo, cuidado primário; na avaliação de EA de causa específica - por exemplo, medicamentos, cirurgias, procedimentos invasivos; em algum profissional de saúde específico - por exemplo, enfermagem; e na abordagem da ocorrência de EA apenas após a alta hospitalar do paciente.

Na revisão bibliográfica, foram utilizadas as seguintes fontes de pesquisa: MEDLINE, COCHRANE, LILACS, SciELO e o banco de teses e dissertações da Coordenadoria de Aperfeiçoamento de Pessoal de Nível Superior CAPES. Foram analisados todos os textos publicados até agosto de 2004. As palavras-chave foram pesquisadas empregando-se o português, o inglês, o francês e o espanhol como idiomas. Foram elas: eventos adversos (adverse event, effets défavorables), revisão de prontuários (chart review, medical record, révision du dossier médical, revisión de historia clínica), estudos médicos (medical practice study, étude médicale, estudio médico de la práctica), hospital (hôpital), complication, iatrogenic disease e a combinação entre elas. As palavraschave e os títulos pesquisados estão sistematizados na Tabela 1, que apresenta o número de artigos encontrados segundo as palavraschave e suas diversas combinações.

A revisão foi realizada pelo primeiro autor do artigo. Ao término da revisão, foram selecionados 129 artigos com estudos baseados na revisão retrospectiva de prontuários que avaliaram a ocorrência de EAs no conjunto das ações dos hospitais. Por questão de espaço, nem todos os 129 artigos estão citados na referência bibliográfica deste trabalho; entretanto, encontramse disponíveis com os autores.

Nos artigos selecionados foram analisados os seguintes aspectos: o tamanho da amostra e a incidência de EAs; a definição de EA; o desenho da avaliação realizada; a proporção de EAs por área clínica, por especialidade, por local de ocorrência, o 
Tabela 1 - Número de artigos encontrados na revisão por palavra-chave e suas combinações segundo a fonte de pesquisa.

Table 1 - Number of articles found by key word, combinations, according to data source.

\begin{tabular}{|c|c|c|c|c|}
\hline \multirow[t]{2}{*}{ FONTES DE PESQUISA } & \multicolumn{4}{|c|}{ NÚMERO DE ARTIGOS } \\
\hline & Total & Excluídos & Sub-Total 1 & Sub-Total $2^{*}$ \\
\hline \multicolumn{5}{|l|}{ MEDLINE } \\
\hline "Adverse events" & 17295 & $\mathbf{N}^{* *}$ & $\mathrm{~N}$ & $\mathrm{~N}$ \\
\hline "Adverse events" hospital & 1479 & 1366 & 105 & 105 \\
\hline "Adverse events" "chart review" & 78 & 67 & 11 & 5 \\
\hline "Adverse events" "medical record" & 53 & 36 & 15 & 3 \\
\hline "Adverse events" "medical practice study" & 373 & 353 & 18 & 8 \\
\hline "Adverse events" complications & 3233 & 3220 & 14 & 4 \\
\hline "Adverse events"“iatrogenic disease" & 120 & 72 & 45 & 4 \\
\hline "Effets défavorables" & 31 & 31 & 0 & 0 \\
\hline “Eventos adversos" & 1 & 1 & 0 & 0 \\
\hline \multicolumn{5}{|l|}{ COCHRANE } \\
\hline "Adverse events" & 303 & 296 & 7 & 0 \\
\hline “Adverse events” hospital & 34 & 34 & 0 & 0 \\
\hline “Adverse events" "chart review" & 2 & 2 & 0 & 0 \\
\hline "Adverse events" "medical record" & 3 & 3 & 0 & 0 \\
\hline “Adverse events”"'medical practice study” & 13 & 13 & 0 & 0 \\
\hline "Adverse events" complications & 49 & 49 & 0 & 0 \\
\hline "Adverse events" iiatrogenic disease" & 2 & 2 & 0 & 0 \\
\hline \multicolumn{5}{|l|}{ LILACS } \\
\hline "Adverse events” & 81 & 81 & 0 & 0 \\
\hline "Effets défavorables" & 0 & 0 & 0 & 0 \\
\hline "Eventos adversos" & 246 & 246 & 0 & 0 \\
\hline \multicolumn{5}{|l|}{ SCIELO } \\
\hline "Eventos adversos" & 25 & 25 & 0 & 0 \\
\hline \multicolumn{5}{|l|}{ Banco de teses da CAPS a partir de 1987} \\
\hline "Eventos adversos" & 16 & 16 & 0 & 0 \\
\hline Total & & & & 129 \\
\hline
\end{tabular}

* Subtotal (1) refere-se aos artigos relacionados ao escopo do estudo. Subtotal (2) refere-se aos artigos relacionados ao escopo do estudo, depois de eliminada a superposição de artigos coincidentes.

* Subtotal (1) refers to articles related to the study scope. Subtotal (2) refers to articles related to study scope, after the overlapping of the same articles was eliminated.

** $\mathrm{N}$ - Refere-se a artigos não pesquisados devido a grande quantidade de referências encontradas.

** Refers to articles not searched due to the large number of references found

momento da ocorrência e de detecção do EA; a gravidade da lesão; e o tempo de permanência no hospital atribuído ao EA..

\section{Resultados}

Os 129 artigos selecionados apresentam 9 estudos sobre a ocorrência de EAs. Esses estudos, que serão descritos e comparados nesta revisão, foram realizados nos EUA - Estados da Califórnia, Nova York, Utah e Colorado -, na Austrália, na Nova Zelândia, no Reino Unido, na França, na Dinamarca e no Canadá.
O estudo pioneiro, The Medical Insurance Feasibility Study $(M I F S)^{10}$, foi realizado pelas California Medical Association e California Hospital Association, por um grupo de médicos legistas. Foi analisada uma amostra de 20.864 prontuários de pacientes internados em 1974, em 23 hospitais do estado da Califórnia. Essa amostra é representativa dos pacientes internados nos hospitais de curta permanência privados e governamentais não federais nesse Estado. A incidência de EAs foi de 4,6 por 100 pacientes (Tabela 2). 
Tabela 2 - Tamanho da amostra, incidência de EAs e proporção de EAs evitáveis nos estudos de avaliação da ocorrência de EAs em hospitais, baseados em revisão retrospectiva de prontuários. Table 2 - Sample size, adverse event (AE) incidence and proportion of preventable hospital AE in studies based on the retrospective review of medical records.

\begin{tabular}{lccc}
\hline Estudo - Data - Local & $\begin{array}{c}\text { Número de } \\
\text { prontuários } \\
\text { na amostra }\end{array}$ & $\begin{array}{c}\text { Incidência de EAS } \\
\text { por } 100 \text { pacientes } \\
\text { internadost }\end{array}$ & $\begin{array}{c}\text { Proporção } \\
\text { de EAs } \\
\text { evitáveis } \dagger\end{array}$ \\
\hline $\begin{array}{l}\text { Mills et al, Califórnia, } \\
\text { 1974. MIFS }\end{array}$ & 20864 & $4,65(4,36-4,94)$ & Não relatada \\
$\begin{array}{l}\text { Brennan et al, Leape et al, } \\
\text { Nova York, 1984. HMPS } \\
\text { Thomas et al, Utah, }\end{array}$ & 30121 & $3,7(3,2-4,2)$ & Negligência* \\
$\begin{array}{l}\text { Colorado, 1992. UCMPS. } \\
\text { Wilson et al Austrália, }\end{array}$ & 14700 & $2,9(2,7-3,1)$ & Negligência * \\
$\begin{array}{l}\text { 1992. QAHCS. } \\
\text { Davis et al, Nova Zelândia, } \\
\text { 1998. AENZS }\end{array}$ & 6579 & $16,6(16,0-17,2) 10,6 \neq$ & 50,3 \\
$\begin{array}{l}\text { Vincent et al, Inglaterra } \\
\begin{array}{l}\text { 1999- 2000. BAES } \\
\text { Baker et al, Canadá, }\end{array}\end{array}$ & 1014 & $11,3(10,4-12,1)$ & $61,6(55,5-67,8)$ \\
$\begin{array}{l}\text { 2000. CAES } \\
\text { Michel et al, França, }\end{array}$ & 3745 & 10,8 & 52,0 \\
$\begin{array}{l}\text { 2002. } \\
\text { Schioler et al, Dinamarca, } \\
\text { 2001. DAES }\end{array}$ & 778 & $7,5(5,7-9,3)$ & $36,9(32,0-41,8)$ \\
\hline
\end{tabular}

* Percentual de eventos devidos à negligência - Brennan et al - 27,6 (22,5 - 32,6), Thomas et al - 32,6 (28,6 - 36,6) em Utah e $27,4(25,0-29,8)$ em Colorado.

* Percentage of events due to negligence - Brennan et al - 27.6 (22.5 - 32.6), Thomas et al - $32.6(28.6$ - 36.6) in Utah and 27.4 (25.0 - 29.8) in Colorado.

† Intervalo de confiança de 95\%/† 95\% Confidence Interval

‡ Incidência obtida quando utilizado o método do UCMPS/キ Incidence obtained when the UCMPS method was used

Cerca de dez anos após o MIFS, foi realizado The Harvard Medical Practice Study $(\text { HMPS })^{9,11,12}$. O método de investigação desse estudo serviu com base para os demais estudos apresentados neste trabalho. Foi analisada uma amostra de 30.121 prontuários de pacientes internados, em 1984, no Estado de Nova York, envolvendo 52 hospitais. Essa amostra é representativa dos pacientes internados nos hospitais de curta permanência privados e governamentais não federais nesse Estado. A incidência de EAs foi de 3,7 por 100 pacientes, sendo um quarto desses devido à negligência. Esses números são muito próximos aos encontrados no MIFS (4,6 por 100 pacientes) alguns anos antes. $\mathrm{O}$ interesse em medir a ocorrência desse fenômeno nos EUA e a busca de soluções preventivas aumentaram com a ampla divulgação desse estudo. Concomi- tantemente à avaliação dos prontuários, foram contatadas companhias de seguro e apuradas 68 mil queixas de má prática médica entre os anos de 1984 e 1989. Esses dados foram cruzados com os dos prontuários. Além disso, um inquérito foi realizado entre os pacientes identificados como tendo sofrido algum EA e foi calculado o impacto econômico decorrente dos EAs ${ }^{13}$, ${ }^{14}$ (Tabela 2).

The Utah Colorado Medical Practice Study (UCMPS) ${ }^{15}$ também foi realizado nos EUA, nos Estados de Utah e Colorado. Foi analisada uma amostra de 14.700 prontuários de pacientes internados, em 1992, nos Estados supracitados, envolvendo 26 hospitais. Essa amostra é representativa dos pacientes internados nos hospitais de curta permanência desses Estados. O desenho da amostra garante a represen- 
tatividade dos hospitais, incluindo os hospitais urbanos e rurais, de ensino ou não, privado lucrativo e não, e público. Hospitais especializados em psiquiatria, reabilitação e tratamento de alcoolismo foram excluídos da amostra. Os hospitais participaram voluntariamente e nenhum se recusou a participar do estudo. A incidência de EAs na amostra foi 2,9 por 100 pacientes, sendo $32,6 \%$ dos casos de EAs em Utah e 27,4\% no Colorado, devido à negligência (Tabela 2).

Na Austrália, também em 1992, foi realizado The Australian Quality Health Care Study (QAHCS $)^{16,17}$. Foi analisada uma amostra aleatória de 14.179 prontuários de pacientes internados, envolvendo 28 hospitais australianos. Essa amostra é representativa dos pacientes internados nos hospitais de curta permanência no país. A incidência de EAs foi de 16,5 por 100 pacientes (Tabela 2). Esse foi o estudo que encontrou a mais alta incidência de EAs entre os estudos analisados. Essa discrepância motivou a realização de um estudo comparando os dados australianos com os dados de Utah e Colorado. Aplicando-se a metodologia do UCMPS nos dados do QAHCS, foram obtidos os seguintes resultados: 10,6 por 100 pacientes na Austrália e 3,2 por 100 no Colorado e em Utah (Tabela 2). A diferença que permaneceu após essa comparação poderia ser explicada por diferenças na qualidade do cuidado, no conteúdo dos prontuários e no comportamento dos médicos revisores ${ }^{16}$. Uma segunda comparação mostrou uma incidência mais próxima entre o UCMPS e QAHCS, no caso dos EAs graves. Nos eventos menos graves, o QAHCS apresentou incidência bem mais elevada. Os autores consideram que o principal fator da discrepância nos resultados entre os estudos poderiam ser os objetivos diferentes de cada um deles - médico legal, no caso americano, e melhoria de qualidade no outro $^{18}$. A partir do QAHCS, os trabalhos passaram a pesquisar não mais a negligência e sim os EAs evitáveis. EA evitável foi definido como um erro no cuidado prestado ao paciente devido a uma falha, individual ou do sistema, em não conformidade com a boa prática ${ }^{18}$. No QAHCS a proporção de EAs evitáveis foi de $50,3 \%$ (Tabela 2).

Em 1998, foi realizado estudo semelhante na Nova Zelândia - Adverse Events in New Zealand Public Hospitals Study $(\text { AENZS })^{19,20}$. Foi analisada uma amostra de 6.579 prontuários de pacientes internados, envolvendo 13 hospitais. Essa amostra é representativa dos pacientes internados nos hospitais de curta permanência com mais de 100 leitos, excluídos os psiquiátricos, de reabilitação e os pacientes-dia. A incidência de EAs foi de 11, 3 por 100 pacientes. No AENZS, a proporção de EAs evitáveis [preventable averse events] foi de 61,6\% (Tabela 2).

Ao final de 1999 e início de 2000, foi desenvolvido o British Adverse Events Study $(B A E S)^{21}$ na Grã Bretanha. Foi analisada uma amostra aleatória de 1.014 prontuários de pacientes internados, entre dezembro de 1999 e fevereiro de 2000, envolvendo 2 hospitais de curta permanência em Londres. A incidência de EAs foi de 10,8 por 100 pacientes. No BAES, a proporção de EAs evitáveis foi de $52 \%$ (Tabela 2 ).

Em 2002, foi realizado um estudo que procurou comparar três métodos para investigar a ocorrência de EAs - métodos prospectivo, retrospectivo e transversal ${ }^{22}$. Foi analisada uma amostra de 778 prontuários de pacientes internados no sudoeste da França, envolvendo 7 hospitais públicos e privados. A amostra restringiu-se aos pacientes internados em enfermarias de clínica médica, clínica cirúrgica e obstetrícia. A incidência de EAs variou em função do método utilizado. O método de revisão retrospectiva de prontuários apontou para uma incidência de EAs de 14,5 em 100 pacientes e a proporção de EAs evitáveis de 27,6\% (Tabela 2). A incidência pelo método retrospectivo foi semelhante ao método prospectivo, porém este identificou uma maior proporção de eventos evitáveis. $\mathrm{O}$ método transversal apresentou um resultado diverso dos demais (9,8 EAs por 100 pacientes) e, apesar de ser o método de estudo menos dispendioso, foi considerado 
de baixa validade para a avaliação de EAs e subestima a incidência de EA, particularmente as mortes decorrentes de EAs ${ }^{22}$.

Na Dinamarca, foi realizado o Danish Adverse Event Study (DAES) ${ }^{23}$. Foi analisada uma amostra de 1.097 prontuários de pacientes internados, obtida do Registro Nacional de Pacientes da Dinamarca, em 2001, envolvendo 17 hospitais. Essa amostra é representativa dos pacientes internados em hospitais de curta permanência no país. A incidência de EAs foi de 9 por 100 pacientes. No DAES, a proporção de EAs evitáveis foi de $40,4 \%$ (Tabela 2).

O último estudo revisado foi The Canadian Adverse Event Study (CAES) ${ }^{24}$. Foi analisada uma amostra de 3.745 prontuários de pacientes internados em 2000, com mais de 18 anos, e que permaneceram internados por mais de 24 horas, em cinco províncias - British Columbia, Alberta, Ontario, Quebec e Nova Scotia. A amostra excluiu os pacientes psiquiátricos e obstétricos. Em cada província, foram selecionados aleatoriamente 1 hospital universitário, 1 grande hospital comunitário e dois pequenos hospitais comunitários. Foram relacionados hospitais que distassem menos de duzentos e cinqüenta quilômetros do Centro de Pesquisa, com pelo menos 1.500 admissões em 2000 e uma emergência aberta 24 horas. Hospitais especializados foram excluídos. A incidência de EAs foi de 7,5 por 100 pacientes. No CAES, a proporção de EAs evitáveis foi de 36,9\% (Tabela 2).

\section{Definições de eventos adversos nos estudos}

O HMPS introduziu o termo EA, definindo-o como lesão [injury] causada pelo cuidado médico e não pela doença de base, que prolongou a estadia do paciente ou resultou numa incapacidade [disability] presente no momento da alta ${ }^{12}$. Dois elementos destacam-se nessa definição. O primeiro é a distinção entre a lesão causada pelo cuidado e a lesão que é conseqüência da evolução da doença de base; o segundo é a especificação do fato da lesão ter sido causada pelo cuida- do prestado na organização médica [medical error]. Esse estudo, que também foi desenvolvido no bojo das discussões sobre o mercado do seguro privado, se preocupa com a questão da negligência, isto é, da responsabilidade individual do médico na ocorrência do $\mathrm{EA}^{11}$. O UCMPS adotou as mesmas definições de EAs e de negligência do HMPS ${ }^{26,27}$. Entretanto, é importante salientar que o termo medical error, muito utilizado na literatura de língua inglesa sobre EAs, na língua portuguesa pode ser erroneamente traduzido como erro do médico. Como bem destaca Lucian Leape, pesquisador americano, medical error é um problema mais sistêmico do que individual ${ }^{25}$.

O QAHCS originou-se da preocupação com a melhoria de qualidade do cuida$\mathrm{do}^{16,18}$. A Australian Patient Safety Foundation considera que EA é sinônimo do termo dano iatrogênico - significando lesão que se origina no cuidado, ao invés de lesão proveniente da doença de base ${ }^{28}$. A partir desse estudo, o termo incapacidade passou a ser mais bem especificado dano temporário ou permanente do paciente, ou morte. Considerou, também, como EA, a lesão sem uma incapacidade caracterizada, mas que tenha levado o paciente ao prolongamento do tempo de permanência no hospital, ou simplesmente perda financeira para o paciente. A definição do QAHCS ${ }^{18}$ - como senso lesão não intencional que resultou em incapacidade temporária ou permanente e/ou prolongamento do tempo de permanência ou morte como conseqüência do cuidado prestado - foi utilizada posteriormente pelo AZENS ${ }^{19}$, BAES $^{21}$, CAES $^{24}$ e pelo estudo francês ${ }^{22}$.

O desenho dos estudos. Em todos os estudos, com exceção do CAES, participaram pacientes de todas as idades. No CAES, indivíduos menores de 18 anos foram excluídos da amostra. Todos os estudos revisados, com exceção do MIFS, basearamse no desenho original do HMPS. Nesse estudo, a avaliação da ocorrência de EA foi realizada por revisão retrospectiva de prontuários, em duas etapas. A primeira 
etapa de rastreamento de EA foi realizada com o emprego de critérios de avaliação explícitos e a segunda, de identificação de EA, com o emprego de critérios de avaliação implícitos. Na fase de rastreamento, sempre que um dos critérios estivesse presente, o prontuário era selecionado para análise na etapa seguinte de avaliação da ocorrência de $\mathrm{EA}^{31}$. Na etapa de avaliação explícita, enfermeiros treinados selecionaram os prontuários de paciente com potencial EA com base em critérios previamente definidos. A maioria dos estudos revisados utilizou 18 critérios de rastreamento, embora nem sempre os mesmos ${ }^{29}$ (Tabela 3). Na segunda fase de avaliação implícita, médicos avaliaram a ocorrência de EAs com o emprego de formulário padronizado e identificaram se estes eram evitáveis, e em alguns estudos se eram devidos à negligência dependendo do enfoque do estudo ${ }^{30}$.

Houve diferenças nos critérios de rastreamento utilizados por cada estudo. O HMPS e o UCMPS coincidiram na maioria dos critérios. Já o CAES e o AENZS coincidiram entre si, mas não em relação ao HMPS e UCMPS. O estudo francês diferiu dos demais, até porque o objetivo desse estudo foi comparar os diferentes métodos para a avaliação da ocorrência de EA. O QAHCS utilizou critérios dos HMPS e UCMPS e influenciou os critérios do CAES e do AENZS (Tabela 3). Os artigos revisados sobre o MIFS, o DAES e o BAES não explicitaram os critérios utilizados.

Os 23 critérios de rastreamento listados na Tabela 3 indicam que houve um aperfeiçoamento destes nos estudos mais recentes. Algumas mudanças merecem destaque: (1) o critério 1, presente no QAHCS, AENZS e CAES, passou a considerar os EAs ocorridos antes da internação índice internação selecionada nos estudos eventos estes não contabilizados nos estudos anteriores - HMPS e UCMPS e (2) o critério 20 - temperatura corporal $>38.3^{\circ} \mathrm{C}$ no dia anterior à alta - presente nos estudos HMPS e UCMPS, que ganhou mais precisão nos estudos QAHCS, AENZS e
CAES, assumindo a denominação de infecção/septicemia hospitalar - critério 19.

A etapa de avaliação implícita foi realizada por médicos revisores, através do emprego de formulários de avaliação estruturados. Esses médicos inicialmente avaliam sua concordância com a presença do critério explícito, que levou a seleção do caso como potencial EA pelo enfermeiro revisor e, posteriormente, avaliam se realmente ocorreu o EA. O HMPS utilizou dois médicos revisores por prontuário. O UCMPS utilizou um médico revisor por prontuário, mas estabeleceu uma supervisão mais rígida exercida por membros da equipe de pesquisa. A partir do QAHCS, todos os demais estudos passaram a realizar a revisão explícita com dois médicos trabalhando em conjunto. Os revisores, para caracterizarem um EA, deveriam constatar a descrição no prontuário de uma lesão causada pelo cuidado e a existência de incapacidade. Caso uma dessas três condições não fosse preenchida, o prontuário era desprezado. Em caso positivo, a avaliação continuava buscando qualificar o EA e a presença ou não de negligência no caso do HMCS e UCMPS, ou se este poderia ter sido evitado, no caso dos demais estudos apresentados nesta revisão. Uma vez identificada a existência de EA, os revisores buscaram definir o local em que ocorreu o EA, o momento da ocorrência, a gravidade da lesão e qual o serviço que foi responsável pelo EA. Esses são os fatores que qualificam o EA.

Os estudos utilizaram diferentes formas de agrupar de EAs. Os artigos que relataram os resultados do QAHCS ${ }^{16,17,18}$ apresentaram a incidência de EAs por Diagnose Related Groups (DRGs), sem a preocupação de agregá-los ainda mais, como em outros estudos. O HMPS e UCMPS ${ }^{9,12}$ agruparam os EAs por áreas clínicas: procedimentos cirúrgicos - EAs por falha na técnica, sangramento pós-operatório, ferida cirúrgica infectada e outra infecção que não de ferida operatória; relacionados a drogas - antibióticos, drogas cardiovasculares, analgésicos, anticoagulantes e outros; relaciona- 
Tabela 3 - Critérios de rastreamento de EA utilizados nos estudos revisados.

Table 3 - AE tracking criteria used in the studies reviewed.

Critério

Estudo*

1. Internação não planejada (incluindo reinternação) como resultado de qualquer $\begin{array}{llllll}1 & 2 & 3 & 4 & 5 & 6\end{array}$ cuidado de saúde prestado durante os 12 meses anteriores à internação índice.

2. Internação não planejada em qualquer hospital durante os 12 meses subseqüentes à alta da internação índice.

3. Ocorrência de internação em pacientes < 65 anos no ano pesquisado.

4. TMP > de percentil 90 do Diagnose Related Group (DRG) em paciente abaixo de 70 anos e percentil 95 em pacientes com 70 anos ou mais.

5. Ocorrência de lesão no paciente durante a internação (inclusive qualquer dano, lesão ou trauma ocorridos durante a internação índice).

6. Reação adversa ao medicamento.

7. Transferência não planejada para unidade de cuidados intensivos.

8. Transferência não planejada para outro hospital de cuidados agudos (excluindo as transferências para exames, procedimentos ou cuidados especializados não disponíveis no hospital de origem).

9. Retorno não planejado à sala de cirurgia.

$X \quad X \quad X \quad X \quad X$

10. Remoção, lesão ou correção não planejada de um órgão ou estrutura durante cirurgia, procedimento invasivo ou parto vaginal.

11. Procedimento cirúrgico "aberto" não planejado após laparoscopia ou admissão após um procedimento invasivo "fechado" ou laparoscópico planejado.

12. Outras complicações do paciente, p.ex. infarto agudo do miocárdio (IAM), acidente vascular cerebral (AVC), embolia pulmonar etc. (incluir quaisquer complicações inesperadas ocorridas durante a internação em referência que NÃO sejam um desenvolvimento normal da doença do paciente ou um resultado esperado do tratamento).

13. Desenvolvimento de déficit neurológico ausente na admissão, mas presente no momento da saída da internação índice (incluem déficits neurológicos relacionados aos procedimentos, tratamentos ou investigações).

14. Óbito inesperado.

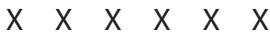

$X \times X \times X$

15. Alta hospitalar inapropriada/planejamento de alta inadequado para a internação índice (exclui alta à revelia).

$\begin{array}{llllll}X & X & X & X & X & X\end{array}$

$X \times \quad X$

$X \times X \times X$

16. Parada cardio-respiratória (com ressuscitação bem sucedida).

17. Lesão ou complicação relacionada com o parto ou gravidez e complicações neonatais.

18. Lesão ou complicação relacionada com o ambulatório de cirurgia ou um procedimento invasivo que resultou na admissão pela emergência.

19. Infecção/septicemia hospitalar (excluir infecções/septicemia ocorridas em menos de 72 horas após a admissão).

20. Temperatura corporal $>38.3^{\circ} \mathrm{C}$ no dia anterior à alta.

$X \quad X \quad X$

21. Insatisfação com o cuidado recebido, documentada no prontuário, ou evidência de queixa apresentada (incluir documentos, queixas documentadas, conflitos entre o paciente/família e profissionais, e alta à revelia).

$\begin{array}{lllllll}X & X & X & X & X & X \\ X & X & X & X & X & X\end{array}$

$X$

$X \quad X$

22. Documentação ou correspondência indicando litígio, seja somente intenção ou ação efetiva.

23. Quaisquer outros eventos indesejados não mencionados acima.

$x \times x \times x$ 
dos a procedimentos médicos, definidos como procedimentos não cirúrgicos; relacionados à demora em efetuar diagnóstico ou diagnóstico incorreto; relacionados a tratamento não medicamentoso inapropriado ou não realizado no tempo necessário; relacionados ao pós parto e ao período neonatal; relacionados à anestesia; a quedas; à fratura, entre outros. No HMPS, foram investigados EAs originados pelo ambiente do cuidado [system]. Os EAs provenientes do ambiente de cuidado foram devidos a equipamento ou material com defeito ou não disponível; comunicação inadequada entre os profissionais; treinamento ou supervisão do médico ou de outro profissional inadequado; demora na prestação de algum serviço (raios-X, teste de laboratório, follow up, visitas etc.); inadequação dos recursos humanos [inadequate staffing]; funcionamento inadequado do hospital (farmácia, serviço de hemoterapia etc.). A proporção da ocorrência de EAs relacionados à cirurgia representou $47,7 \%$ do total de EAs no HMPS e $44 \%$ no UCMPS 9 (Tabela 4). A proporção de EAs relacionada ao uso de medicamentos foi a mesma nos dois estudos, cerca de 19,\% (Tabela 4). A proporção desses dois tipos de EAs somados aproxima-se de 70\%. No HMPS, as proporções mais altas de EAs, devidos à negligência, foram originadas por incorreção no diagnóstico e no tratamento não medicamentoso - 75,2\% e 76,8\%, respectivamente, e no UCMPS 93,8\% e 56,8\%, respectivamente. No UCMPS, 65,8\% dos EAs devidos a queda foram considerados negligência. No HMPS, a incorreção no diagnóstico foi responsável por 8,1\% dos EAs considerados muito graves, e o ambiente do cuidado contribuiu com 3,3\% dos EAs.

Os artigos que apresentaram os resultados do AENZS e do CAES ${ }^{19,20,24}$ agruparam os EAs de forma semelhante ao HMPS e UCMPS: procedimentos cirúrgicos; relacionados aos medicamentos; aos procedimentos médicos; ao diagnóstico; a tratamento não medicamentoso; ao ambiente do cuidado, dentre outros. No CAES, além dos já citados, os EAs foram atribuídos a anestesia; a fraturas e a acidentes obstétricos. No item ambiente do cuidado, além dos citados anteriormente no HMPS, estão incluídos os EAs provenientes da inexistência de protocolos clínicos ou da falha na sua aplicação.

Os EAs devidos a procedimentos cirúrgicos e os medicamentos também foram os mais freqüentes no CAES e AENZS $36,6 \%$ e $57,7 \%$, respectivamente (Tabela 4 ). O AENZS apresentou a maior proporção de EAs causados por falhas no ambiente de cuidados $-24 \%$.

Os estudos relacionaram a incidência de EAs por especialidade. A proporção de EAs detectados no BAES $^{21}$ em medicina geral foi de $9,2 \%$; na cirurgia geral, $16,2 \%$; na obstetrícia, $4 \%$; e na ortopedia, $14,4 \%$. A proporção de EAs evitáveis por especialidade foi bem mais alta na medicina geral e na obstetrícia - $76 \%$ e $71 \%$ - do que na cirurgia geral e na ortopedia $-43 \%$ e $33 \%$, respecti-

Tabela 4 - Proporção de EA segundo a área clínica de ocorrência do evento por estudo. Table 4 - Proportion of AE by clinical area of occurrence.

\begin{tabular}{lcccc}
\hline Área clínica / Estudo & HMPS & UCMPS & AENZS & CAES \\
\hline Cirúrgico & 47,7 & 44,9 & 24,3 & 34,1 \\
Medicamentoso & 19,4 & 19,3 & 12,3 & 23,6 \\
Sub total & 67,1 & 64,2 & 36,6 & 57,7 \\
Procedimento médico & 7,0 & 13,5 & 7,7 & 12 \\
Demora ou incorreção no diagnóstico & 8,1 & 6,9 & 8,0 & 10,5 \\
Demora ou incorreção no tratamento & 7.5 & 4,3 & 8,4 & 7,3 \\
Outros* & 10,7 & 11,1 & 15,3 & 3,1 \\
Ambiente de cuidados & 3,3 & - & 24,0 & 9,4 \\
\hline
\end{tabular}

* EA por queda, fraturas, obstétricos, neonatais, anestésicos. /* AE due to fall, fractures, obstetrics, neonatal, anesthetics 
vamente. A proporção de EAs por especialidade, encontrada no AENZS, em medicina geral foi de $35,7 \%$ e em cirurgia, $57,5 \%$.

O momento do cuidado em que ocorreu o EA pode ter sido antes, durante ou após a internação índice e pode ter sido detectado antes, durante ou após a mesma. O momento de ocorrência e de detecção foi relatado em três estudos - HMPS, CAES e AENZS ${ }^{11-}$ 13,19,20,24. Nesses três estudos, a maior parte dos EAs ocorreram e foram detectados durante a internação índice - $76 \%$ do total de EAs no HMPS, $57 \%$ no CAES e $52 \%$ no AENZS. A ocorrência de EAs antes e detectadas durante a internação índice foi de 13\% no HMPS, 31\% no CAES e 35\% no AENZS. A ocorrência de EAs durante e a detecção após a internação índice foi de $11 \%$ no HMPS, $12 \%$ no CAES e $13 \%$ no AENZS.

O local de ocorrência do EA também foi apresentado nos estudos. Os artigos sobre o AENZS ${ }^{19,20}$ classificaram o local de ocorrência de EAs na internação índice como tendo sido dentro ou fora do hospital. Foi considerado fora do hospital todo cuidado prestado fora do ambiente do hospital - consultório do médico, casa do paciente e nursing home. No AENZS, mais de $80 \%$ dos pacientes sofreram a lesão causada pelo cuidado, dentro do hospital (Tabela 5). Os artigos sobre o HMPS e o UCMPS ${ }^{11-}$ ${ }^{14,32}$ especificaram quais locais dentro e fora do hospital ocorreram os EAs. Tal como no AENZS, no HMPS e no UCMPS, a incidência de EAs dentro do hospital foi de mais de $80 \%$ (Tabela 5). A sala cirúrgica e a enfermaria/quarto foram os locais onde mais EAs ocorreram - $41 \%$ e $26,5 \%$ no HMPS e $39,5 \%$ e $21,6 \%$ no UCMPS. Foram nos consultórios médicos que ocorreu a maior parte dos EAs fora do ambiente do hospital $7,7 \%$ e $7,0 \%$ no HMPS e no UCMPS, respectivamente. O MIFS considerou apenas EAs que ocorreram dentro do hospital. Pacientes que sofreram EAs fora do hospital não fizeram parte desse estudo.

A gravidade da lesão resultante do EA foi apresentada nos artigos sobre o MIFS, o HMPS, o UCMPS, o CAES e o AENZS ${ }^{10-}$ 14,19,20,24,32. A presença de incapacidade foi avaliada pelos médicos revisores no momento da alta. Esta foi classificada diferentemente nos vários estudos. No HMPS a classificação foi definida como: (1) dano mínimo, com recuperação em 1 mês; (2) dano moderado, com recuperação de 1 a 6 me-

Tabela 5 - Proporção de EA segundo o local de ocorrência do evento.

Table 5 - Proportion of AE by place of occurrence

\begin{tabular}{|c|c|c|c|c|}
\hline Local de ocorrência / Estudo & HMPS & UCMPS & MIFS & AENZS \\
\hline No Hospital & 81,2 & 83,8 & 100 & 80,4 \\
\hline Sala de cirurgia & 41,0 & 39,5 & 71,8 & \\
\hline Enfermaria /quarto & 26,5 & 21,6 & 12,0 & \\
\hline Sala de parto e de pré-parto & 2,8 & 6,5 & 4,6 & \\
\hline Sala de cateterização cardíaca & 0,9 & 4,2 & - & \\
\hline Unidade de cuidados intensivos & 2,7 & 3,5 & 0,6 & \\
\hline Sala de procedimentos & - & 3,4 & 0,7 & \\
\hline Sala de emergência & 2,9 & 3,0 & - & \\
\hline Unidade ambulatorial & 0,9 & - & - & \\
\hline Radiologia & 2,0 & - & 5,8 & \\
\hline Outros & 1,7 & 2,2 & 2,1 & \\
\hline Fora do hospital & 18,8 & 16,2 & & 19,6 \\
\hline Consultório médico & 7,7 & 7,0 & & \\
\hline Em casa & 2,7 & 3,4 & & \\
\hline Hospital dia & 1,4 & 1,2 & & \\
\hline Nursing home & 0,9 & 0,6 & & \\
\hline Outros & 6,2 & 4,1 & & \\
\hline Total & 100,00 & 100,00 & 100,00 & 100,00 \\
\hline
\end{tabular}


ses; (3) dano moderado com recuperação de 6 meses a 1 ano; (4) dano permanente, com recuperação com d" $50 \%$ de incapacidade; (5) dano permanente, com recuperação com e" $50 \%$ de incapacidade; e (6) morte. O UCMPS utilizou as categorias emocional, insignificante, temporário menor e maior, permanentes menor, significante e maior, grave e morte. O AENZS classificou como: (1) dano mínimo < 1 mês; (2) dano moderado, entre 1 mês e 1 ano; (3) dano permanente d•50\%; (4) dano permanente e•50 \%; e (5) morte. O CAES acrescentou à classificação do AZENS a categoria "nenhum”. Como a definição de EA no CAES caracteriza EA como lesão causada pelo cuidado que resulta em incapacidade, morte ou prolongamento da estadia do paciente no hospital, a categoria "nenhum" representa especificamente o prolongamento dos dias de permanência no hospital.

Os artigos sobre o CAES e AENZS ${ }^{19,20,24}$ apresentaram os tempos médios de permanência dos pacientes no hospital, com e sem EAs. No CAES, considerando apenas os hospitais universitários, a permanência de pacientes sem EAs foi em média 7,8 dias e dos pacientes com EAs foi de 17,7 dias. No AENZS, a média foi de 3 dias para os pacientes sem EAs e 5 dias para os pacientes com EAs. No CAES, considerando apenas os hospitais universitários, e no AENZS, a média de dias extras de permanência no hospital por paciente foi de 6,2 e 9,5 dias, respectivamente. No BAES ${ }^{21}$, a média de dias extras por paciente foi de 8,5 dias.

\section{Discussão}

Os nove estudos pesquisados neste trabalho, que utilizaram revisão retrospectiva de prontuários para analisar a ocorrência de EAs no conjunto das ações dos hospitais, apresentaram diferença significativa na incidência de EAs. Enquanto no HMPS e no UCMPS a incidência oscilou entre 2,9 e 3,7 por 100 pacientes, no QAHCS chegou a 16,6 por 100 pacientes. Mesmo aplicando a metodologia do UCMPS nos dados do QAHCS, a diferença continuou expressiva -
10,6 por 100 pacientes na Austrália e 3,2 por 100 no Colorado e em Utah. Essa disparidade de resultados poderia ser explicada por diferenças na qualidade do cuidado, diferença no conteúdo dos prontuários e diferença no comportamento dos médicos revisores ${ }^{16}$. No caso dos EAs graves, a incidência mostrou-se mais próxima no UCMPS e QAHCS, enquanto nos eventos menos graves 0 QAHCS apresentou incidência bem mais elevada. Baker e cols. consideram que os estudos americanos tinham uma preocupação de caráter médico legal, enquanto nos demais o eixo de preocupação estava vinculado a ações de melhoria de qualidade, explicando a diferença entre os estudos ${ }^{18,24}$. Estes últimos estudos incluíram entre seus objetivos a melhoria de desempenho dos processos de cuidado aos pacientes. Sob essa lógica, os médicos revisores valorizaram os EAs de menor gravidade, contribuindo para o aumento da sua incidência. Em dois formulários de avaliação com critérios implícitos - HMPS e CAES -, existem diferenças importantes na abordagem e na precisão dos itens de avaliação. Baker e cols. apontam que a inclusão do critério "Internação não planejada (incluindo reinternação) como resultado de qualquer cuidado de saúde prestado durante os 12 meses anteriores à internação índice" ajuda a explicar em alguma escala as disparidades na ocorrência de EAs verificadas nos diversos estudos. O CAES limitou em maiores de 18 anos os indivíduos selecionados para pesquisa, sem que o motivo tenha sido explicitado nos artigos pesquisados. Esse mesmo estudo excluiu pacientes obstétricas, consideradas pelos autores de baixo risco de EA.

Embora os estudos tenham conservado uma estrutura semelhante - uma fase de rastreamento com critérios explícitos e uma segunda fase de avaliação com critérios implícitos, algumas diferenças na metodologia entre os estudos foram observadas nesta revisão. Houve diferenças nos critérios de rastreamento utilizados por cada estudo. O UCMPS utilizou apenas 1 médico revisor, enquanto todos os demais utilizaram 2 médicos revisores que avalia- 
ram conjuntamente o mesmo prontuário. Há evidência de que a confiabilidade dos métodos de avaliação de prontuários médicos pode ser melhorada pela combinação da avaliação de dois ou mais médicos ${ }^{33}$.

Este trabalho de revisão não teve a intenção apenas de conhecer a realidade da situação dos EAs, mas também avaliar as potencialidades e limitações do método de revisão retrospectiva de prontuários. Esta revisão indicou que o método pode ser útil em estudos em países que não possuem informações eletronicamente disponíveis, como o Brasil. Segundo a Sociedade Brasileira de Informática em Saúde (SBIS), não existe no país nenhum hospital que utilize integralmente o prontuário eletrônico ${ }^{35}$. No Brasil, o uso de banco de dados administrativos também não é adequado para a avaliação de EAs devido à limitada disponibilidade de informação sobre co-morbidade. No Sistema de Informações Hospitalares do Sistema Único de Saúde - SIH-SUS - existe campo para registro de apenas 1 diagnóstico secundário. Outras abordagens, como a apuração de caráter voluntário com informação fornecida pelo próprio profissional, esbarra ainda na cultura predominante de que o erro é uma vergonha e não um mecanismo de aprendizado.
A ocorrência de EAs é considerada um problema de importância internacional. A OMS criou em 2002 um Grupo de Trabalho com o objetivo de estudar metodologias para avaliar de forma sistemática a segurança do paciente nos serviços de saúde e definiu um programa para 2005 - World Alliance for Patient Safety - criando um suporte www.who.int/patientsafety, acessado em 12/01/2005 - para o desenho, planejamento e monitoramento de ações para segurança do paciente, integradas entre os países ${ }^{34}$.

A preocupação de que o cuidado à saúde não deve ser a causa de um agravo ao paciente vem desde Hipócrates - Primum non necere é um postulado. Os resultados dos estudos investigados neste trabalho de revisão contrariam esse postulado hipocrático. Entender este fenômeno é um importante passo para pensar políticas que garantam a segurança dos pacientes que procuram assistência em hospitais.

\section{Agradecimentos}

Agradecemos aos revisores da Revista Brasileira de Epidemiologia e à auxiliar de pesquisa Priscilla Marques pelos apontamentos e correções feitas durante a revisão deste artigo, contribuindo bastante com os autores na elaboração do texto final.

\section{Referências}

1. World Health Organization. Patient Safety: Rapid Assessment Methods for Estimating Hazards. Genebra; 2003.

2. Kohn LT, Corrigan JM, Donaldson MS, McKay T, Pike KC. To err is human. Washington, DC: National Academy Press; 2000.

3. Gallotti, R. M. D. Eventos adversos e óbitos hospitalares em serviço de emergências clínicas de um hospital universitário terciário: um olhar para a qualidade da atenção [tese de doutorado]. São Paulo: Faculdade de Medicina da Universidade de São Paulo; 2003.

4. Kritek PB. Rethinking the critical care environment: luxury or necessity? AACN Clin Issues 2001; 336-44.

5. Codman EA. A study in hospital efficiency. Illinois: Oakbrook Terrace; 1996.
6. Barr DP. Hazards of modern diagnosis and therapy-the price we pay. JAMA 1955; 159: 1452-6.

7. Murff HJ, Patel VL, Hripcsak G, Bates DW. Detecting adverse events for patient safety research: a review of current methodologies. JAMIA 2003; 36:131-43.

8. Corrigan JM, Kohn LT, Donaldson MS, Maguire SK, Pike KC. Crossing the quality chasm: a new health system for the $21^{\text {st }}$ century. Washington, DC: National Academy Press; 2001.

9. Studdert DM, Brennan TA, Thomas EJ. What have we learned since the Harvard Medical Practice Study? In: Rosenthal MM, Sutcliffe, Kathleen M, eds. Medical Error: what do we know? What do we do? EUA: Jossey-Bass; 2002. p. 3-34.,

10. Mills DH. Medical insurance feasibility study. A technical summary. West J Med 1978; 128: 360-5. 
11. Brennan TA, Hebert L, Lair NM, Lawthers AG, Thorpe KE, Leape LL et al. Hospital characteristics associated with adverse events and substandard care. JAMA 1991; 265: 3265-9.

12. Leape LL, Brennan TA, Laird N, Lawthers AG, Localio AR, Barnes BA et al. The nature of adverse events in hospitalized patients. Results of the Harvard Medical Practice Study II. N Engl J Med 1991; 324: 377-84.

13. Brennan TA, Localio AR, Laird NM. Reliability and validity of judgments concerning adverse events and negligence. Med Care 1989; 27: 1148-58.

14. Studdert DM, Brennan TA. No-fault compensation for medical injuries: the prospect for error prevention. JAMA 2001; 286: 217-23.

15. Gawande AA, Thomas EJ, Zinner MJ, Brennan TA. The incidence and nature of surgical adverse events in Colorado and Utah in 1992. Surgery 1999; 126: 66-75.

16. Wilson RM, Runciman WB, Gibbert RW, Harrison BT, Newby L, Hamilton JD. The Quality in Australian Health Care Study. Med J Aust 1995; 163: 458-71.

17. Wilson RM, Runciman WB, Gibbert RW, Harrison BT, Hamilton JD. Quality in Australian Health Care Study. Med J Aust 1996; 164: 754.

18. Runciman WB, Webb RK, Helps SC. A comparison of iatrogenic injury studies in Australia and the USA II: context, methods, casemix, population, patient and hospital characteristics. Int J Qual Health Care 2000; 12:379-88.

19. Davis P, Lay-Yee R, Schug S, Briant R, Scott A, Johnson S et al. Adverse events regional feasibility study: indicative findings. NZMed J 2001; 114: 203-5.

20. Davis P, Lay-Yee R, Scott A, Briant R, Schug S. Acknowledgement of a "no fault" medical injury: review of patients' hospital records in New Zealand. Br Med J 2003; 326: 79-80.

21. Vincent C, Neale G, Woloshynowych M. Adverse events in British hospitals: preliminary retrospective record review. Br Med J 2001; 322: 517-9.

22. Michel P, Quenon JL, Sarasqueta AM, Scemama O. Comparison of three methods for estimating rates of adverse events and rates of preventable adverse events in acute care hospitals. Br Med J 2004; 328: 199-202.

23. Schioler T, Lipczak H, Pedersen BL, Mogensen TS, Bech KB, Stockmarr A et al. Danish Adverse Event Study, Incidence of adverse events in hospitals. A retrospective study of medical records. Ugeskr Laeger 2002; 164: 4377-9.
24. Baker GR, Norton PG, Flintoft V, Blais R, Brown A, Cox J et al. The Canadian Adverse Events Study: the incidence of adverse events among hospital patients in Canada. CMAJ 2004; 170: 1678-86.

25. Unruh L. Trend in Adverse Events in Hospitalized Patients. J. Healthcare Quality 2002; 24(5): 4-10

26. Thomas EJ, Lipsitz SR, Studdert DM, Brennan TA. The reliability of medical record review for estimating adverse event rates. Ann Intern Med 2002; 136:812-6.

27. Thomas EJ, Studdert DM, Runciman WB. A comparison of iatrogenic injury studies in Australia and the USA I: context, methods, casemix, population, patient and hospital characteristics. Int J Qual Health Care 2000; 12:371-8.

28. Runciman, W. B., Roberts, R., and Madden, R. An iatrogenic injury classification. World Health Organization (WHO), Cardiff, Wales, UK. 1999.

29. Localio AR, Weaver SL, Landis JR, et al. Identifying adverse events caused by medical care: degree of physician agreement in a retrospective chart review. Ann Intern Med 1996; 125:457-64.

30. Goldman R. Development of a Veterans Administration occurrence screening program. QRB Qual Ver Bull 1989; 15:315-9.

31. Leape LL, Bates DW, Cullen DJ, Cooper J, Demonaco HJ, Gallivan T et al. Systems analysis of adverse drug events. ADE Prevention Study Group. JAMA 1995; 274: 35-43.

32. Thomas EJ, Studdert DM, Burstin HR, Orav EJ, Zeena T, Williams EJ et al. Incidence and types of adverse events and negligent care in Utah and Colorado. Med Care 2000; 38: $261-71$.

33. Camacho LAB, Rubi HR. Reliability of Medical Audit in Quality Assessment of Medical Care. Cad Saúde Pública 1996; 12: 85-93.

34. World Health Organization.World Alliance for Patient Safety: forward programme. Genebra; 2005.

35. Sociedade Brasileira de Informática em Saúde (SBIS) 2004. Disponível em www.sbis.org.br (acessado em 17-92004.)

Recebido em: 05/05/05 Versão reformulada reapresentada em: 23/08/05 Aprovado em: 12/09/05 